\title{
A multi-point iterative method for solving nonlinear equations with optimal order of convergence
}

\begin{abstract}
In this study, a three-point iterative method for solving nonlinear equations is presented. The purpose is to upgrade a fourth order iterative method by adding one Newton step and using a proportional approximation for last derivative. Per iteration this method needs three evaluations of the function and one evaluation of its first derivatives. In addition, the efficiency index of the developed method is $\sqrt{ } 48 \approx 1.682$ which supports the Kung-Traub conjecture on the optimal order of convergence. Moreover, numerical and graphical comparison of the proposed method with other existing methods with the same order of convergence are given.
\end{abstract}

Keyword: Multi-point iterative methods; Simple root; Order of convergence; Kung and Traub's conjecture; Efficiency index 